Anima Nagar, V. Kannan and S. P. Sesha Sai, Department of Mathematics \& Statistics, University of Hyderabad, Hyderabad 500046, India. e-mail:

sm028s@uohyd.ernet.in, vksm@uohyd.ernet.in, and

sm020s@uohyd.ernet.in

\title{
PROPERTIES OF TOPOLOGICALLY TRANSITIVE MAPS ON THE REAL LINE
}

\begin{abstract}
We prove that every topologically transitive map $f$ on the real line must satisfy the following properties:

(1) The set $C$ of critical points is unbounded.

(2) The set $f(C)$ of critical values is also unbounded.

(3) Apart from the empty set and the whole set, there can be at most one open invariant set.

(4) With a single possible exception, for every element $x$ the backward orbit $\left\{y \in \mathbb{R}: f^{n}(y)=x\right.$ for some $n$ in $\left.\mathbb{N}\right\}$ is dense in $\mathbb{R}$.
\end{abstract}

\section{Introduction}

Topologically transitive maps on intervals in the real line, and their dynamical properties have been well-studied. See [4], [5], [2], [7] and [6]. In [9] it has been proved that every such map should have a dense set of periodic points and should be chaotic. The aim of this paper is to establish some more properties that every topologically transitive map on $\mathbb{R}$ should possess. These results can be used

(a) to supplement a result of [7] and

(b) to show that the examples constructed in [1] are among the simplest possible ones.

Key Words: Topologically transitive maps, critical points, critical values, invariant set. Mathematical Reviews subject classification: $54 \mathrm{H} 20$.

Received by the editors October 26, 2000 
We start by recalling some well-known technical terms. If $f$ is a continuous self map on an interval $I$ in the real line $\mathbb{R}$, then the pair $(I, f)$ is called a dynamical system. If $n$ is a positive integer, $f^{n+1}$ denotes the composite of $f^{n}$ with $f$, where $f^{1}$ denotes $f$. A subset $A$ of $I$ is said to be invariant, if $f(A) \subset A$. The system $(I, f)$ is said to be topologically transitive if whenever $V$ and $W$ are nonempty open subsets of $I$, there is a positive integer $n$ such that $f^{n}(V) \cap W$ is nonempty; this happens if and only if there is $x$ in $I$ such that its orbit $\left\{f^{n}(x): n \in \mathbb{N}\right\}$ is dense in $I$. In all these, $I$ can be bounded or unbounded or even the whole $\mathbb{R}$. Hereafter "topologically transitive" will be briefly referred to as "transitive". An element $x$ in $I$ is said to be a critical point of $f$ if in every neighborhood $V$ of $x$ there are distinct $y, z$ in $V$ such that $f(y)=f(z)$. We denote by $C$ the set of all critical points.

Now we are ready to state our theorems.

Theorem 1. Let $(\mathbb{R}, f)$ be such that $C$ is bounded. Then there is a nontrivial compact interval $I$ such that either $I$ or its complement $I^{c}$ or a component of $I^{c}$ is invariant.

Corollary. If $(\mathbb{R}, f)$ is transitive, then $C$ is unbounded. In particular, $f$ cannot be a polynomial.

Remark. When $C$ is unbounded, there are examples to show that the conclusion of Theorem 1 can fail.

Theorem 2. Let $(\mathbb{R}, f)$ be surjective. Then $C$ is bounded if and only if $f(C)$ is bounded.

Corollary. If $f$ is transitive, then the set $f(C)$ of critical values is unbounded.

Remark. There are examples of transitive $(\mathbb{R}, f)$ where $C$ and $f(C)$ are bounded below. See [1].

Theorem 3. Let $(\mathbb{R}, f)$ be transitive. Then there are at most three subsets of $\mathbb{R}$ that are open and invariant; namely the empty set, the whole set and if the third exists, it is the complement of a singleton.

Corollary. Let $(\mathbb{R}, f)$ be transitive. Then the backward orbit of $x$, i.e.,

$$
\left\{y \in \mathbb{R}: f^{n}(y)=x \text { for some } n \in \mathbb{N}\right\}
$$

is dense in $\mathbb{R}$, for all $x$ with at most one exception.

Remark. There are examples to show that this kind of exceptional point may or may not exist. 


\section{Proofs of the Main Results}

The following eight elementary observations about a continuous self-map of $\mathbb{R}$ will be used later in the proofs:

(1) Every transitive map on $\mathbb{R}$ has a dense connected range, and therefore the map is surjective.

(2) If $x$ is in the closure of the set $C$ of all critical points of $f$, then every neighborhood $V$ of $x$ is a neighborhood of some critical point, and therefore $f$ is not one-one on $V$. Thus $C$ is closed.

(3) Every point of local maximum or local minimum should be a critical point; but the converse is not true.

(4) If there is no critical point of $f$ in an interval of $\mathbb{R}$, then $f$ is strictly monotonic on that interval; if $C$ is empty, then $f$ is strictly monotonic on the whole of $\mathbb{R}$.

(5) If $C$ is bounded, then there is a left ray $(-\infty, a]$ and a right ray $[b, \infty)$ on both of which $f$ is strictly monotonic.

(6) If $f$ is not onto, then the range of $f$ is contained in a proper (left or right) ray.

(7) If $f$ is transitive, and if $A$ is an invariant set, then either $A$ or $A^{c}$ is dense. In particular, no left ray, no right ray and no nontrivial compact interval can be invariant, if $f$ is transitive on $\mathbb{R}$.

(8) A transitive $f$ on $\mathbb{R}$ cannot admit a bounded invariant set with nonempty interior.

Theorem 1. Let $f: \mathbb{R} \rightarrow \mathbb{R}$ be continuous and let the set $C$ of critical points of $f$ be bounded. Then there exist $p<q$ in $\mathbb{R}$ such that either $[p, q]$ or its complement or $(-\infty, p]$ or $[q, \infty)$ is invariant.

Proof. If there are two fixed points $x<y$ with no critical point between them, then $f$ is monotonic on $[x, y]$ and the interval $[x, y]$ is invariant, proving the theorem in this case. If this case does not hold, then the boundedness of $C$ implies that the set $F$ of all fixed points is also bounded; the set $f(C)$ of critical values must also be bounded, because $f$ is continuous and $C$ is bounded; therefore there are numbers $c<d$ such that

$$
C \cup f(C) \cup F \subset[c, d] .
$$


We consider three subcases.

Case (a): Let $f(x)>x$ for some $x>d$. Then $f(x)>x$ for all $x>d$ because there are no fixed points and no critical points $>d$. In this subcase, the right ray $[d, \infty)$ is invariant.

Case (b): Similarly, if $f(x)<x$ for some $x<c$, then the left ray $(-\infty, c]$ is invariant.

Case (c): If neither Case (a) nor Case (b) holds, then $f(x)>x$ for all $x<c$; and $f(x)<x$ for all $x>d$. Consider three subcases of this subcase. We know $f$ is monotonic on $(-\infty, c]$ and $[d, \infty)$.

Case (i): Let $f$ be increasing on both $(-\infty, c]$ and $[d, \infty)$. Then we claim that $[c, d]$ is invariant. For this, let $a$ and $b$ respectively be the infimum and supremum of $C$. Then $f([a, b]) \subset$ any interval containing $f(C)$; this is because, all local maximum values and local minimum values should be in $f(C)$. Also, if $c \leq x \leq a$ then $f(c) \leq f(x) \leq f(a)$ (since $f$ has to be increasing on $[c, a]$ also, because of absence of critical points) and $c \leq f(c)$ and $f(a) \in[c, d]$. These imply that $f([c, a]) \subset[c, d]$. Similarly, $f([b, d])$ is also $\subset[c, d]$. Combining these, we have that $[c, d]$ is invariant.

Case (ii): Of the two rays $(-\infty, c]$ and $[d, \infty)$, let $f$ be increasing on one of them and decreasing on the other. Then $f$ is not onto. [If $f$ is increasing on $(-\infty, c]$ and decreasing on $[d, \infty)$, then $f$ is bounded above; in fact

$$
\sup f(\mathbb{R})=\sup f([c, d]) .
$$

Similarly, in the other case, $f$ is bounded below.] Therefore the range of $f$ is an invariant ray.

Case (iii): $f$ is decreasing both on $(-\infty, c]$ and $[d, \infty)$. Then we may assume

$$
\lim _{x \rightarrow \infty} f(x)=-\infty \text { and } \lim _{x \rightarrow-\infty} f(x)=\infty,
$$

since in the other cases, $f$ fails to be onto, and therefore invariant rays are easily available. Because of these limits at $\pm \infty$, there exists $x_{0}>d$ such that $f\left(x_{0}\right)<c$. Then on the ray $\left[x_{0}, \infty\right), f \cdot f$ is increasing. Suppose for some $y \geq x_{0}$, it is true that $f(f(y)) \geq y$. Then it is easy to prove that

$$
[y, \infty) \cup(-\infty, f(y)]
$$

is invariant. Suppose such $y$ does not exist. Then $f(f(y))<y$ holds for all $y \geq x_{0}$. Now we claim that $\left[f\left(x_{0}\right), x_{0}\right]$ is invariant. To prove this, we first note that $f$ is decreasing on $(-\infty, a]$ and also on $[b, \infty)$. If $t$ lies between $f\left(x_{0}\right)$ and $a$, then

$$
f(t) \leq f\left(f\left(x_{0}\right)\right)<x_{0}
$$


also

$$
f(b) \geq f(a) \geq c \geq f\left(x_{0}\right) .
$$

Similarly, if $t$ lies between $b$ and $x_{0}$, then $f(b)$ lies between $f\left(x_{0}\right)$ and $x_{0}$. Again if $t$ is in $[a, b]$, then

$$
f(b) \in[c, d] \subset\left[f\left(x_{0}\right), x_{0}\right] .
$$

Thus we have

$$
f\left(\left[f\left(x_{0}\right), a\right] \cup[a, b] \cup\left[b, x_{0}\right]\right) \subset\left[f\left(x_{0}\right), x_{0}\right] .
$$

Thus $\left[f\left(x_{0}\right), x_{0}\right]$ is invariant.

Thus in all cases we get a nontrivial compact interval, or a ray, or the complement of a compact interval, as an invariant set.

Theorem 2. Let $f: \mathbb{R} \rightarrow \mathbb{R}$ be continuous and surjective. If the set $C$ of critical points is unbounded, then $f(C)$ is also unbounded.

Proof. First we prove an elementary result; Let $a$ and $b$ be two critical points with $a<b$. Then the maximum value of $f$ on $[a, b]$ is a critical value. If $f(a)$ or $f(b)$ is the maximum value, then this is easily seen. If not, let $a<c<b$ be such that $f(c) \geq f(t)$ for all $t$ in $[a, b]$. Then $f$ fails to be one-one on $(c-\epsilon, c+\epsilon)$ for all $\epsilon>0$. Therefore $c$ is a critical point and thus $\max f([a, b])$ is a critical value.

If $f(C)$ were bounded, let $f(C) \subset[m, M]$. Let

$$
A=\cup\{[a, b]: a<b \text { are elements in } C\} .
$$

Then $f(A)$ is also $\subset[m, M]$ by our first step. Consider three cases.

Case 1: If $C$ is neither bounded above nor bounded below, then $A=\mathbb{R}$. In this case, the range of $f$ is $\subset[m, M]$, a contradiction.

Case 2: If $C$ is bounded below, let $a=\inf C$. Then $A=[a, \infty)$ (since Case 1 does not hold). Then $f$ is strictly monotonic on $(-\infty, a]$. Therefore the range of $f$ is contained in

$$
\text { either }(-\infty, f(a)] \cup[m, M] \text { or }[m, M] \cup[f(a), \infty) .
$$

In either case $f$ fails to be surjective.

Case 3: If $C$ is bounded above, we argue similarly. 
Lemma. Let $(\mathbb{R}, f)$ be transitive. Then $f^{-1}(x)$ has empty interior, for every $x$ in $\mathbb{R}$.

Proof. Let if possible, $V=$ nonempty interior of $f^{-1}(x)$, for some $x$ in $\mathbb{R}$. We claim that $x$ should be a periodic point. By transitivity, $\exists n \in \mathbb{N}$ such that $f^{n}(V) \cap V$ is nonempty. But since $f(V)=\{x\}, f^{n}(V)$ is a singleton $\subset V$. Then $f^{n+1}(V)=\{x\}$, thus $f^{n}(x)=x$. It follows that

$$
V \cup(\text { the finite orbit of } x),
$$

is an invariant set. This set is not dense, because its image under $f$ is finite. This contradicts transitivity.

Corollary. Let $(\mathbb{R}, f)$ be transitive. Let $A$ be a subset of $\mathbb{R}$ with nonempty interior. Let $n$ be a positive integer. Then $f^{n}(A)$ also has nonempty interior.

Proof. Let $W$ be a nontrivial open interval $\subset A$. Then $f^{n}(W)$ is connected. It cannot be a singleton, as can be proved by induction on $n$, using the above lemma. Therefore $f^{n}(W)$, and hence $f^{n}(A)$, contains a nonempty open interval.

Theorem 3. Let $(\mathbb{R}, f)$ be transitive. Let $A$ be an invariant set with nonempty interior. Then $A^{c}$ is at most a singleton.

Proof. Let $V$ be a nonempty open interval $\subset A$. We shall prove that

$$
B=\cup_{n=0}^{\infty} f^{n}(V)
$$

(which is a subset of $A$ ) omits at most one real number. Let if possible $a<b$ be two real numbers not in $B$. The transitivity of $f$ ensures that some $f^{n}(V)$ meets the open interval $(a, b)$; in fact $f^{n}(V) \cap(a, b)$ has nonempty interior, by the above corollary. Thus $B$ has a nontrivial bounded component, say $B_{0}$ which is contained in $(a, b)$. Again by transitivity, $\exists m \in \mathbb{N}$ such that $f^{m}\left(B_{0}\right)$ meets the interior of $B_{0}$. But since $f^{m}\left(B_{0}\right)$ is a connected subset of $B$ and since $B_{0}$ is a component of $B$, it follows that $f^{m}\left(B_{0}\right) \subset B_{0}$. But then $\cup_{k=0}^{m} f^{k}\left(B_{0}\right)$ is a bounded invariant subset with nonempty interior, contradicting the transitivity of $f$.

Corollary. Let $(\mathbb{R}, f)$ be transitive. Then there is at most one $x$ in $\mathbb{R}$ whose backward-orbit

$$
B_{x}=\left\{y \in \mathbb{R}: f^{n}(y)=x \text { for some } n \text { in } \mathbb{N}\right\}
$$

fails to be dense in $\mathbb{R}$. 
Proof. If $B_{x}$ is not dense, then let $V=\left(\bar{B}_{x}\right)^{c}$. Then $V$ is an invariant set with nonempty interior. Therefore by Theorem $3, V^{c}$ is at most a singleton. Therefore $B_{x}=\{x\}$.

Suppose now $x<y$ are two points such that $B_{x}=\{x\}$ and $B_{y}=\{y\}$. Then $f(x)=x ; f(y)=y$. Also, if for some $t$ in $[x, y], f(t)<x$, then by intermediate value theorem some element in $[t, y]$ should be mapped to $x$, contradicting $B_{x}=\{x\}$. Similarly no element of $[x, y]$ goes beyond $y$. Thus $[x, y]$ is invariant, again contradicting the transitivity.

Corollary. Let $(\mathbb{R}, f)$ be transitive. Then all but one of the equivalence classes $[x]$ must be dense in $\mathbb{R}$.

Corollary. Let $(\mathbb{R}, f)$ be transitive, then the set of all pre-critical points is dense.

Proof. There are many critical points and all but possibly one of them, must have dense backward- orbit.

Corollary. Let $(\mathbb{R}, f)$ be transitive. Let $J$ be any nonempty open interval in $\mathbb{R}$. Then there is a positive integer $n$ such that $f^{n}$ is not one-one on $J$.

Proof. By the previous corollary, $J$ contains a pre-critical point $y$ such that some $f^{n}(y)$ is a critical point. Then $f^{n+1}$ is not one-one on $J$.

\section{Remarks and Examples}

In Theorem 1 we proved that the boundedness of $C$ implies the existence of two distinct points $p>q$ in $\mathbb{R}$ such that either

$$
(-\infty, p] \text { or }[q, \infty) \text { or }[-\infty, p) \cup(q, \infty)
$$

is invariant. If instead of $\mathbb{R}$, our underlying set is $\mathbb{R}^{+}=[0, \infty)$, the same methods yield a more elegant conclusion as stated below:

Theorem 4. Let $f:[0, \infty) \rightarrow[0, \infty)$ be a continuous map such that the set $C$ of critical points is bounded [equivalently such that $f$ is one-one on a right ray]. Then there exists $d>0$ such that either $[0, d]$ or $[d, \infty)$ is $f$-invariant.

Proof. As in the proof of Theorem 1, there is some $d>0$ such that

$$
C \cup f(C) \cup F \subset[0, d] .
$$

On $[d, \infty) f$ is strictly monotonic, without fixed points. 
If $f$ is decreasing there, then $f$ is bounded above, and if $c=$ supremum of the range of $f$, then $[0, c]$ is invariant. (In fact, the supremum of range of $f$ can be proved to be equal to $d$ in this case.)

If $f$ is increasing on $[d, \infty)$, we consider two cases, according as the graph of $f$ is above the diagonal or below the diagonal on $[d, \infty)$. In the former case, $f(x)>x$ for all $x>d$ and therefore $[d, \infty)$ is invariant. In the latter case, $f(x)<x$ for all $x<d$ and we claim that $[0, d]$ is invariant.

If $x \leq \sup C$, then $f(x) \leq d$ since $f(C) \subset[0, d]$ by the choice of $d$.

If $\sup C<x \leq d$, then $f$ is increasing on $[x, \infty)$ and therefore $f(x) \leq f(d) \leq d$. Combining these, we obtain $f([0, d]) \subset[0, d]$.

Remark. Thus a nontrivial connected invariant set is guaranteed by the boundedness of $C$, for all continuous self maps of $\mathbb{R}^{+}$. It follows that all transitive maps on $\mathbb{R}$ or $\mathbb{R}^{+}$must have an unbounded set of critical points. We can state it in a better way, after observing that

(i) transitivity is preserved by topological conjugacy and

(ii) every noncompact interval in $\mathbb{R}$ is homeomorphic to either $\mathbb{R}$ or $\mathbb{R}^{+}$.

Corollary. Let $J$ be a noncompact interval in $\mathbb{R}$ and let $f: J \rightarrow J$ be transitive. Then the set of critical points of $f$ is noncompact (and therefore infinite).

In particular, no polynomial can be transitive on $J$. Contrast this with the known result that on compact intervals, there are polynomial functions that are transitive. A well-known example is the logistic function $4 x(1-x)$ on $[0,1]$.

Remark. Now it is natural to ask if the conclusion of Theorem 1 holds for all continuous maps on the real line. The answer is in the negative. The conclusion of Theorem 1 need not hold, when $C$ is unbounded. Given any positive integer $n$, one can construct a continuous self-map on $\mathbb{R}$ satisfying the property that every invariant set $A$, such that neither $A$ nor $A^{c}$ is dense, should have exactly $n$ connected components. For $n=3$, an example is described below. 
First, $f$ is defined on integers by prescribing

$$
\left\{\begin{array}{lll}
f(0)=3 & f(1)=4 & f(2)=10 \\
f(3)=6 & f(4)=7 & f(5)=10 \\
f(6)=0 & f(7)=1 & f(8)=10 \\
f(2 n)=2 n+2 & \text { for all } n \geq 4 & \\
f(2 n+1)=-2 n & \text { for all } n \geq 4 & \\
f(-n)=f(n) & \text { for all } n \geq 1 &
\end{array}\right.
$$

and then $f$ is defined at other points by demanding that $f$ is linear between any two consecutive integers. For this $f$, the set

$$
[0,1] \cup[3,4] \cup[6,7] \text { is an invariant set. }
$$

Every invariant set $A$ such that neither $A$ nor $A^{c}$ is dense, must be contained in this set, and must have three connected components.

Remark 3. The following definition is taken from [7]. A piecewise continuous piecewise monotonic map $f$ on an interval is said to be strongly transitive, if for every nonempty open set $V$ there is a positive integer $n$ such that $\cup_{k=0}^{n} f^{k}(V)=$ the whole domain. In [7], the last corollary of that paper is stated as under:

Every strongly transitive map $f$ on the unit interval (with or without each end-point) having finitely many maxima and minima, is topologically conjugate to a uniformly piecewise linear map.

Now we make three comments on this statement, when we make the extra assumption that $f$ is continuous:

(1) On a noncompact interval, every transitive continuous map must have infinitely many maxima and minima (and therefore certain parts of the hypothesis are never satisfied).

(2) No continuous map on a noncompact interval can be strongly transitive.

(3) Every continuous piecewise monotonic transitive map on a compact interval is strongly transitive (and thus there is no distinction between transitivity and strong transitivity among these maps).

Of these, the first one follows immediately from the corollary under Theorem 4 . The second can be proved easily by combining the following facts: 
(i) Every noncompact interval is homeomorphic to $\mathbb{R}$ or $\mathbb{R}^{+}$;

(ii) If $f$ is any continuous self-map of $\mathbb{R}$ or $\mathbb{R}^{+}$and if $V$ is a bounded open subset of that space, then $\cup_{k=0}^{n} f^{k}(V)$ is also bounded for each $n$ in $\mathbb{N}$,

The third result is given in [3].

Remark. The simplest set that can appear as the set $C$ of critical points of a transitive $f$ on $\mathbb{R}$, is (by Corollary to Theorem 1 ) an infinite discrete closed set like $\mathbb{N}$ or $\mathbb{Z}$. That is why, the examples in [1] are among the simplest possible examples.

\section{References}

[1] Anima Nagar and S. P. Sesha Sai, Some classes of transitive maps on $\mathbb{R}$, Jour. of Anal., 8 (2000), 103-111.

[2] Annalisa Cranneli, The role of transitivity in Devaney's definition of chaos, Amer. Math. Monthly, 102 (1995), 788- 791.

[3] E. Coven and I. Mulvey, Transitivity and the centre for maps of the circle, Ergodic Theory and Dynamical Systems, 6 (1986), 1-8.

[4] R. L. Devaney, An introduction to chaotic dynamical systems, AddisonWesley, 1989.

[5] H. Holmgren, A first course in discrete dynamical systems, SpringerVerlag, 1996.

[6] Kolyada and Snoha, Some aspects of topological transitivity - a survey, Grazer Math. Ber., 334 (1997), 3-35.

[7] M. Parry, Symbolic dynamics and transformations of the unit interval, Trans. Amer. Math. Soc., 122 (1966), 368- 378.

[8] S. P. Sesha Sai, Symbolic dynamics for complete classification, Ph. D. Thesis, University of Hyderabad, (2000).

[9] M. Vellekoop and R. Berglund, On intervals, transitivity = chaos, Amer. Math. Monthly, 101 (1994), 353-355. 